\title{
openheart Coronary risk of patients with valvular heart disease: prospective validation of CT-Valve Score
}

\author{
Rasmus Bo Hasselbalch (D) , ${ }^{1,2}$ Mia Marie Pries-Heje, ${ }^{1}$ \\ Sarah Louise Kjølhede Holle, ${ }^{1}$ Thomas Engstrøm, ${ }^{3}$ Merete Heitmann, ${ }^{4}$ \\ Frants Pedersen, ${ }^{3}$ Morten Schou, ${ }^{1,5}$ Hans Mickley, ${ }^{6}$ Hanne Elming, ${ }^{7}$ \\ Rolf Steffensen, ${ }^{8}$ Lars Koeber, ${ }^{3}$ Kasper Iversen ${ }^{1,2}$
}

\begin{abstract}
- Additional material is published online only. To view please visit the journal online (http://dx.doi.org/10.1136/ openhrt-2020-001380).

To cite: Hasselbalch RB, Pries-Heje MM,

Kjølhede Holle SL, et al. Coronary risk of patients with valvular heart disease: prospective validation of CTValve Score. Open Heart 2020;7:e001380. doi:10.1136/ openhrt-2020-001380
\end{abstract}

Received 6 July 2020 Revised 18 August 2020 Accepted 2 September 2020

Check for updates

\section{(C) Author(s) (or their} employer(s)) 2020. Re-use permitted under CC BY-NC. No commercial re-use. See rights and permissions. Published by BMJ.

For numbered affiliations see end of article.

Correspondence to Dr Rasmus Bo Hasselbalch; rasmus.bo.hasselbalch@ regionh.dk

\section{ABSTRACT}

Objective To prospectively validate the CT-Valve score, a new risk score designed to identify patients with valvular heart disease at a low risk of coronary artery disease (CAD) who could benefit from multislice CT (MSCT) first instead of coronary angiography (CAG).

Methods This was a prospective cohort study of patients referred for valve surgery in the Capital Region of Denmark and Odense University Hospital from the 1 February 2015 to the 1 February 2017. MSCT was implemented for patients with a CT-Valve score $\leq 7$ at the referring physician's discretion. Patients with a history of CAD or chronic kidney disease were excluded. The primary outcome was the proportion of patients needing reevaluation with CAG after MSCT and risk of CAD among the patients determined to be low to intermediate risk. Results In total, 1149 patients were included. The median score was 9 (IQR 3) and $339(30 \%)$ had a score $\leq 7$. MSCT was used for 117 patients. Of these $29(25 \%)$ were reevaluated and $9(7.7 \%)$ had CAD. Of the 222 patients with a score $\leq 7$ that did not receive an MSCT, $14(6 \%)$ had significant CAD. The estimated total cost of evaluation among patients with a score $\leq 7$ before implementation was $€ 132093$ compared with $€ 79073$ after, a 40\% reduction. Similarly, estimated total radiation before and after was $608 \mathrm{mSv}$ and $362 \mathrm{mSv}$, a 41\% reduction. Follow-up at a median of 32 months (18-48) showed no ischaemic events for patients receiving only MSCT.

Conclusion The CT-Valve score is a valid method for determining risk of CAD among patients with valvular heart disease. Using a score $\leq 7$ as a cut-off for the use of MSCT is safe and cost-effective.

\section{INTRODUCTION}

Valvular heart disease (VHD) is a common disease affecting about $2.5 \%$ of the population. ${ }^{1}$ Although as many as one-third of patients are ineligible for surgical treatment, ${ }^{2}$ in most of the patients that are eligible, properative evaluation of possible coronary artery disease $(\mathrm{CAD})$ is recommended. ${ }^{34}$ The gold standard for this evaluation is invasive coronary angiography (CAG) with fractional flow reserve. Recently multislice CT (MSCT)

\section{Key questions}

What is already known about this subject?

- Evaluation for coronary artery disease using coronary angiography is recommended before operations for valvular heart disease. For low-risk patients, multislice CT is an alternative. The CTValve score has been constructed on retrospective cohorts to identify these patients.

What does this study add?

- In this study, we confirm the validity of the CT-Valve Score, a simple clinical risk score of coronary disease for patients with valvular heart disease.

How might this impact on clinical practice?

- The CT-Valve score can be used clinically, and about $30 \%$ of patients could receive a multislice CT. This could save about $40 \%$ of the cost and radiation dosage of evaluating these patients.

has emerged as a less expensive, logistically more available and non-invasive alternative. ${ }^{5}$ Though highly sensitive, the specificity of MSCT remains low, thus a positive scan needs reevaluation by CAG. For this reason, MSCT is recommended as an alternative for CAG only in patients with low to intermediate risk of CAD. ${ }^{3}$ However, there is no established way of determining this risk among patients with VHD.

Recently a clinical risk score, the CT-Valve score, was developed for this purpose using retrospective data of a combined 4796 patients scheduled for surgery for VHD. ${ }^{6}$ Our aim in this study was to prospectively validate the use of this risk score with a primary endpoint of needed reevaluations with CAG after MSCT and risk of CAD among the patients determined to be low to intermediate risk. 


\section{METHODS}

From the 1 February 2015 clinicians in the Region Zealand and the Capital Region of Denmark were encouraged to use MSCT as a gatekeeper for CAG among patients scheduled for surgery for VHD with a low risk of CAD. This was defined as a CT-Valve score of $\leq 7$. Patients with prior ischaemic heart disease (IHD) or chronic kidney disease (CKD) stage IV or worse were not recommended MSCT. A positive or equivocal scan led to reevaluation with CAG. Odense University Hospital implemented the same strategy on the 1 September 2015.

To assess the performance of the CT-Valve score, we conducted this prospective cohort study. Every patient undergoing MSCT or CAG before valve surgery in the Capital Region and the Region of Zealand were registered in a database (the Web-PATS database). From this database as well as electronic patient files, we assessed the patients' risk factors and results of their diagnostic tests. Significant CAD was defined as a stenosis $>70 \%(>50 \%$ for the left main coronary artery) or with a fractional flow reserve $\leq 0.80$. To ensure the safety of the approach, we followed the patients who only received MSCT through electronic patient records after surgery.

\section{The CT-Valve score}

The CT-Valve score is a score of known risk factors of CAD—age, sex, hypertension, hyperlipidaemia, diabetes mellitus, smoking, extracardiac arteriopathy (ECA) as well as left ventricular ejection fraction (LVEF) $<30 \%$ and aortic valve disease. ${ }^{6}$ On implementation of the MSCT first approach, clinicians at all invasive centres were educated on the use of the CT-Valve score and received posters and pocket cards (online supplemental figure 1). In addition, the investigators were in regular contact with key clinicians at each referring centre to remind them to use the score.

\section{Outcome measures}

The primary outcome measure of this study was the reevaluation rate of MSCT. We estimated about one-third of patients would have a score of $\leq 7$ and that this would be associated with a risk of CAD of about $10 \%$ based on a prior study. ${ }^{6}$ Based on an earlier observational study, the reevaluation rate was estimated to be about 2.5 times the prevalence of CAD. ${ }^{7}$ This was primarily due to equivocal results and technical difficulties such as motion artefacts and blooming artefacts caused by coronary calcifications.

Secondary outcome measures included the predictive ability of the CT-Valve score for CAD, the estimated radiation dosage and a basic estimate of the cost-effectiveness of the approach. The radiation dosage was compared for the registered values in the database. The radiation dosage of a CAG was estimated as a product of the dosearea product registered in the database multiplied with a conversion factor for the chest $(\mathrm{k}=0.22 \mathrm{mSv} / \mathrm{mGy} \times$ $\mathrm{cm}^{2}$ ) based on the National Radiological Protection Board tables. ${ }^{8}$ To give a basic estimate of the estimate cost-effectiveness, we initially performed a literature

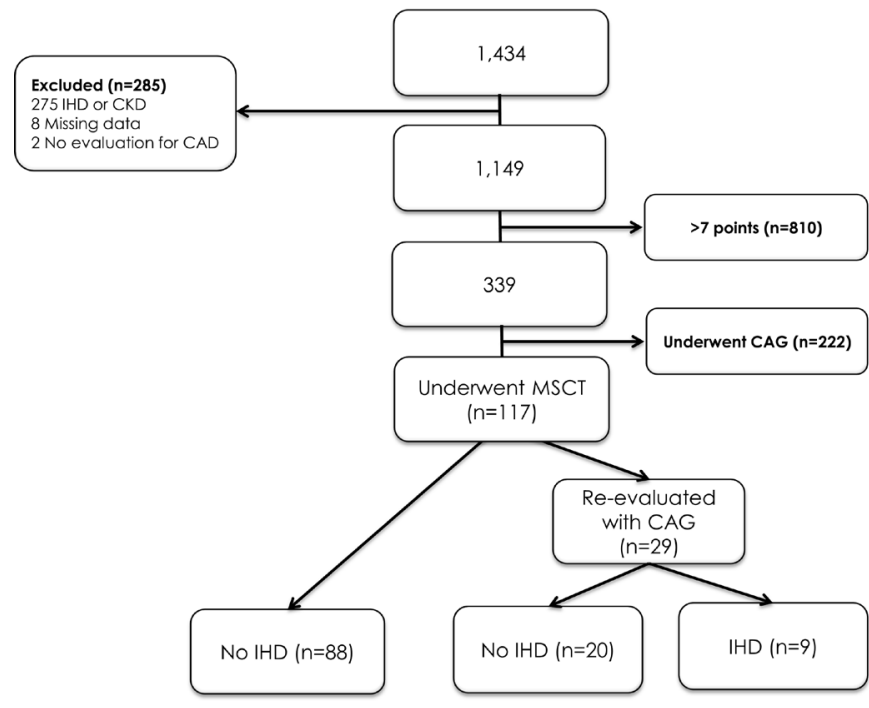

Figure 1 CONSORT diagram. CAG, coronary angiography; $\mathrm{CAD}$, coronary artery disease; CKD, chronic kidney disease; CONSORT, Consolidated Standards of Reporting Trials; IHD, ischaemic heart disease; MSCT, multislice CT.

review and identified prices for CAG and MSCT from seven different countries, the USA, ${ }^{9}$ UK, ${ }^{10}$ Denmark, ${ }^{11}$ Germany, ${ }^{12}$ Australia, ${ }^{13}$ Korea ${ }^{14}$ and Sweden. ${ }^{15}$ The mean cost was for MSCT $€ 396$ and $€ 1129$ for CAG.

\section{Statistics}

Continuous variables are presented with mean (SD) or median IQR and categorical variables as number (n) and percentages $(\%)$. Baseline characteristics were visually inspected for normality and compared using Fisher's exact test or Welch two-sample t-test as appropriate. We used receiver operating characteristics (ROC) to calculate the area under the curve (AUC) as a measure of the predictive ability of the CT-Valve score. Statistical programming was performed in R V.3.5.2 using RStudio V.1.0.136. ${ }^{16}$

\section{Patient and public involvement}

There was no patient or public involvement in the design, or conduct, or reporting, or dissemination plans of our research.

\section{RESULTS}

From the 1 February 2015 to the 1 February 2017, a total of 1434 patients were registered. Of these, two $(0.1 \%)$ patients received no evaluation for CAD because they were considered low risk. We further excluded patients with prior IHD and CKD as well as patients with missing data resulting in a cohort of 1149 patients (figure 1). Of the patients included there were $2(0.2 \%)<40$ years with no cardiovascular risk factors. Among all patients included 302 of 1149 (26\%) had significant CAD on the evaluation before surgery.

The median CT-Valve score for all patients was 9 (IQR $3)$. Figure 2 shows the patient distribution according to the CT-Valve score and proportion of patients with 


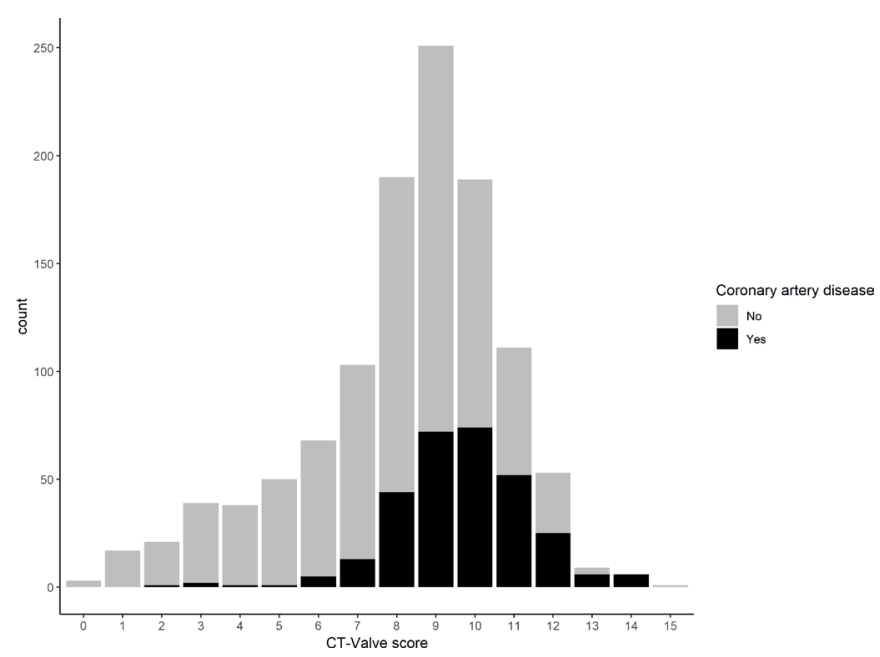

Figure 2 CT-Valve score and the proportion of patients with significant coronary artery disease.

significant CAD. The ROC curve for prediction of CAD is shown in online supplemental figure 2, the AUC was $0.72(95 \%$ CI 0.69 to 0.75$)$. A total of 339 (30\%) patients had a score $\leq 7$ points, table 1 shows the baseline characteristics of patients divided at this cut-off. Patients with a score $\geq 8$ were older by a mean of 15 years and had a higher prevalence of hypertension, hyperlipidaemia, smoking, ECA, LVEF $<30$ as well as aortic valve pathology (all $\mathrm{p}<0.01$ ). Only female gender was not significantly different between the two groups. Among the patients with a score $\leq 7$ points $23(7 \%)$ had $\mathrm{CAD}$, while among patients with as core $\geq 8279(34 \%)$ had a CAD $(p<0.001)$. At the cut-off of 7 points the sensitivity of the CT-Valve score for identifying patients with significant CAD was $92 \%$, the specificity was $37 \%$, the positive predictive value $34 \%$, and negative predictive value was $93 \%$.

\begin{tabular}{|c|c|c|c|}
\hline CT-Valve score & $\begin{array}{l}\leq 7 \\
(n=339)\end{array}$ & $\begin{array}{l}\geq 8 \\
(n=810)\end{array}$ & $P$ value \\
\hline Female, n (\%) & $145(43)$ & $296(37)$ & 0.06 \\
\hline Age, mean (SD) & $60.6(11.3)$ & $76.2(7.1)$ & $<0.001$ \\
\hline Hypertension, n (\%) & $101(30)$ & $611(76)$ & $<0.001$ \\
\hline Hyperlipidaemia, n (\%) & $83(25)$ & $494(63)$ & $<0.001$ \\
\hline Smoking, n (\%) & $131(39)$ & $490(63)$ & $<0.001$ \\
\hline ECA, n (\%) & $1(0.3)$ & $62(7.7)$ & $<0.001$ \\
\hline LVEF <30, n (\%) & $7(2.1)$ & $57(7.2)$ & $<0.001$ \\
\hline \multicolumn{4}{|l|}{ Valve pathology } \\
\hline Aortic valve pathology, $n(\%)$ & $179(53)$ & $718(89)$ & \\
\hline Mitral valve pathology, $\mathrm{n}(\%)$ & $143(42)$ & $78(9.6)$ & \\
\hline Other, n (\%) & $17(4.9)$ & $14(1.7)$ & $<0.001$ \\
\hline
\end{tabular}

$\mathrm{n}(\%)$; age mean (SD), smoking defined as active or past smoker. ECA, extracardiac arteriopathy; LVEF, left ventricular ejection fraction.
Of the 339 patients with a score $\leq 7$ points, $117(35 \%)$ underwent MSCT as initial evaluation for CAD. Online supplemental table 1 shows comparison of baseline characteristics of those who received a scan and those who did not. The patients in the MSCT group where younger (mean difference 6.2 years, $\mathrm{p}<0.001$ ) had a lower risk of hyperlipidaemia $(p=0.046)$ and had a lower CT-Valve score (mean difference $0.9, \mathrm{p}<0.001$ ). However, there was no significant difference in the prevalence of CAD with 9 $(8 \%)$ among the MSCT group having CAD compared with $14(6 \%)$ among the no MSCT group $(\mathrm{p}=0.65)$. Reevaluation with CAG were performed on 29 of the 117 patients $(25 \%)$ and $9(7.7 \%)$ had significant CAD giving a rate of reevaluation to risk of CAD of $29 / 9$ or 3.2. Patients who only received MSCT were followed through electronic records. At a median follow-up time of 32 months (range 18-48) since initial evaluation no patients had any ischaemic events. There were no patients lost to follow-up.

There were Agatston scores available for 73 of the 117 MSCT scans. The median score was 8 (IQR 0-88) and $32(44 \%)$ had a score of 0 . In four patients the Agatston score was so high the clinician decided to forego the MSCT (score of 464, 603, 495 and 799) of these two had significant CAD on a subsequent CAG. For one patient, the clinician decided to re-evaluate an MSCT described as negative with CAG because of an Agatston score of 17 . The patient did not have significant CAD.

The approach of MSCT-first for a score $\leq 7$ was highly cost-effective. For the 117 patients sent to MSCT we estimated a total cost of $€ 79073$, including the cost of the 29 reevaluations. Compared with the estimated price of $€ 132093$ for the same patients receiving a CAG this was savings of $€ 53020$, corresponding to $40 \%$ of the prior cost of evaluating this group $(\mathrm{p}<0.001)$. The estimated cost of all 1149 included patients receiving a CAG would have been $€ 1297221$. Compared with this price, the $€ 53$ 020 saved by 117 patients receiving an MSCT saved 4\% of cost. However, if all 339 patients with a CT-Valve score $\leq 7$ points had received an MSCT first, with the same reevaluation rate as we observed, the savings would have been $€ 164816$ corresponding to a $12 \%$ reduction in cost.

Likewise, the approach led to a lowering of the total radiation dose used per patient from a mean of $6.9 \mathrm{mSv}$ for all patients receiving a CAG to $4.0 \mathrm{mSv}$ ( $\mathrm{p}=0.004$ ) for patients in the MSCT group, including the radiation from reevaluations with $\mathrm{CAG}$. The mean radiation dose of an MSCT was $1.8 \mathrm{mSv}$ (range $0.52-10.8 \mathrm{mSv}$ ). Using the mean dosage estimates among low-risk patients the total radiation saved was $249 \mathrm{mSv}$ corresponding to $41 \%$ of the original dose. The mean contrast dose used for an MSCT was $78 \mathrm{~mL}$ (range $40-230 \mathrm{~mL}$ ) which was slightly higher than the mean contrast use among lowrisk patients undergoing CAG which was $68 \mathrm{~mL}$ (range $20-400), p=0.03$. Using these estimates the approach lead to about $30 \%$ increase in the total contrast use among low-risk patients.

Exploring the potential for different cut-offs for MSCT, we found that at a re-evaluation rate similar to the one 

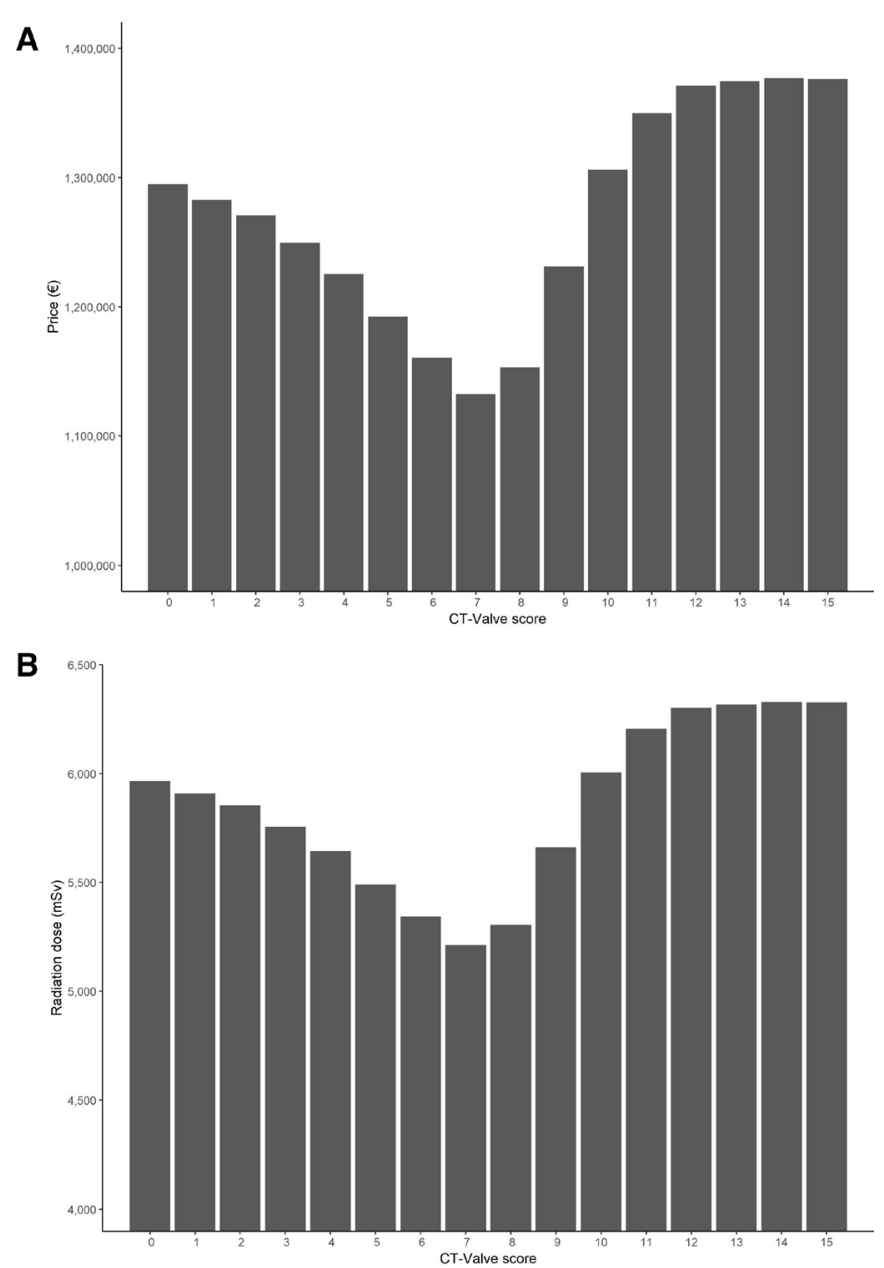

Figure 3 (A, B) Exploration of cutoffs using the average re-evaluation rate of 3.2 derived from our data and the mean price and radiation dose. (A) shows the price of using each point of the score as cut-off for MSCT on all included patients in the study in $€$, (B) shows the corresponding estimated dose of radiation used in $\mathrm{mSv}$. MSCT, multislice CT.

we experienced in this study (3.2) raising the cut-off to 8 would not have been cost-effective, as this would have cost $9.2 \%$ more than just using CAG. In contrast, of the 22 patients with a score of 7 who received an MSCT, 8 $(36 \%)$ were reevaluated which corresponded to an estimated savings of $29 \%$ of the original cost. Figure 3A,B shows the estimated price and radiation dose of all evaluations, both CAG and MSCT, for all included patients in the study using each step of the score as a cut-off value. This clearly shows the cut-off of $\leq 7$ points to be the most cost-effective choice both in terms of radiation and cost.

Complications were rare for both CAG and MSCT. A minor reaction (vomiting) was registered for 1 MSCT patient $(0.9 \%$ of patients receiving MSCT). Serious adverse event for CAG were registered for five patients (0.5\% of patients receiving CAG). Of these four were dissections, one of the Aorta, one of the right coronary artery, one of the brachial artery and one of the iliac artery. Further, six patients $(0.6 \%)$ had a bleeding complication, one of which was a large haematoma $(>10 \times 10 \mathrm{~cm})$.

\section{DISCUSSION}

The CT-Valve score can predict the risk of CAD among patients with VHD. Patients with a CT-Valve score $\leq 7$ have a low risk of CAD. For these patients MSCT is a safe, costeffective alternative to CAG. Thus, we recommend the use of the CT-Valve score to determine the risk of CAD among patients with VHD.

Current guidelines from the European Society of Cardiology and the American College of Cardiologists and the American Heart Association have coronary evaluation of patients referred for valve surgery as a $1 \mathrm{C}$ recommendation. ${ }^{34}$ MSCT is suggested as an alternative rule-out procedure to CAG among patients with a low or low to intermediate risk of CAD. This strategy is supported by data from a recent meta-analysis that confirms that a strategy with the use of MSCT as a rule-out for CAD is safe. ${ }^{5}$ Consistent with this, in this study there were no adverse events associated with undiscovered ischaemia at a median follow-up of 32 months among the patients who only received an MSCT, suggesting this approach is safe.

Guidelines have no recommended strategies to identify patients at low to intermediate risk of CAD. Therefore, the practical approach to the selection of patients that might benefit from a strategy of MSCT as a gatekeeper to CAG is to use CT-Valve score $\leq 7$ excluding patients with a history of CAD or CKD.

A large cohort study from the USA estimated the prevalence of valve disease (moderate and severe) to $2 \%$ corresponding to about 5 million adults. ${ }^{17}$ This number is expected to rise in the coming decades as the population continues to grow older. In the same cohort they estimated that as many as one in eight over the age of 75 in the USA have clinically significant valve disease. ${ }^{17}$ The latest data from the Society of Thoracic Surgeons showed that 72862 patients in the USA received a valve repair or replacement in the year $2017 .{ }^{18}$ Based on our calculations showing that about one-third of patients were at low risk and of a $40 \%$ savings in cost and $41 \%$ in radiation dose among these patients this would result in a yearly savings of approximately 3-9 million dollars and $12000-36000 \mathrm{mSv}$ of radiation dependent on how large a percentage of these patients were sent to MSCT, with the low end corresponding to the $35 \%$ of patients with a CT-Valve score $\leq 7$ receiving an MSCT as seen in this study and the high end being $100 \%$.

At the implementation of the CT-Valve score we chose a conservative cut-off of 7 points as suitable for MSCT even though our calculations on cost-effectiveness suggested a cut-off as high as 9 as feasible. ${ }^{6}$ The reason for this was primarily safety concern, as an earlier study had shown that the sensitivity of MSCT significantly dropped among patients at higher risk of CAD. ${ }^{19}$ Based on a prior retrospective analysis we estimated that about one-third of patients would have a score of $\leq 7$ and that this would be associated with a risk of CAD of about $10 \%{ }^{6}$ Further, we speculated that there would be about 2.5 times as many reevaluations as patients with significant CAD. This was 
extrapolated from a small observational study. ${ }^{7}$ In this study, the reevaluation rate turned out to be more than three re-evaluations per patient with significant CAD. At least one of these procedures were performed even though the MSCT showed no significant CAD. This probably illustrates the clinicians' fear of sending a patient to surgery without absolute certainty that they had no missed CAD. However, the data from this study are reassuring, as none of the patients receiving only MSCT had an ischaemic event during the follow-up.

Fortunately, adverse events were rare in this study with less than $1 \%$ of patients experiencing a serious adverse event during CAG. However, the character of these events highlights the risk of invasive procedures with four patients experiencing dissections of major arteries including one of the Aorta. Bleeding complications were less frequent $(0.6 \%)$ than in earlier studies where it is estimated that $4 \%-7 \%$ of patients experienced vascular complications. ${ }^{20}$ Smaller vascular complications could be under-reported in the database, although a systematic audit of a similar Danish database suggested unreported adverse events were rare. ${ }^{21} 22$ Only one patient had a minor reaction to the contrast in the MSCT.

This study had several limitations. First, this was an observational study, and thus by definition cannot determine whether MSCT is better than CAG among patients with a low CT-Valve score. However, as the study was designed to evaluate the usefulness of the CT-Valve score by examining the number of reevaluations needed and the cost-effectiveness associated with this, randomisation was deemed unnecessary. Further, no patients receiving only MSCT had an adverse event associated with myocardial ischaemia in the follow-up period which highlights the diagnostic accuracy of MSCT among low-risk patients.

Another limitation was that two thirds of the patients with a score $\leq 7$ underwent CAG as the primary investigation. There were multiple reasons for this. First, as MSCT was a new recommendation doctors at referral centres tended to choose CAG out of habit. Furthermore, some patients were not suitable for an MSCT, for example, patients with atrial fibrillation with uncontrollable heart rate. Lastly, for patients needing more urgent evaluation for CAD, CAG was often the fastest way as waiting times in Denmark are often higher for MSCT than CAG.

The basic cost-effectiveness analysis reported as a secondary outcome was very limited in scope, and a future study looking specifically at the cost of each approach in a systematic fashion is needed to determine the costeffectiveness of using the CT-Valve score.

As patients with a score $\geq 8$ did not receive an MSCT this study cannot determine the value of MSCT in this group. However, the high risk of $\mathrm{CAD}$ in this group suggests that MSCT might be of limited use. Further studies could explore the use of a higher cut-off point of the CT-Valve score to see if this is as cost-effective. Similarly, the use of other ischaemic tests was not explored in this study. Unfortunately, we did not have Agatston score results for most patients and thus could not reanalyse the score with this measure included. A future approach for lowrisk patients could include functional imaging tests, such as rubidium- 82 positron emission tomography imaging. However, MSCT is generally viewed as the most costeffective gate keeper. ${ }^{23}$

\section{CONCLUSION}

The CT-Valve score is a valid method in determining the risk of CAD among patients evaluated prior to surgery for VHD. At a CT-Valve score $\leq 7$ points, using an MSCT-first approach to evaluation is safe and cost-effective.

\section{Author affiliations}

${ }^{1}$ Department of Cardiology, Herlev Hospital, Herlev, Denmark

${ }^{2}$ Department of Emergency Medicine, Herlev Hospital, Herlev, Denmark

${ }^{3}$ Department of Cardiology, Copenhagen University Hospital, Rigshospitalet, Copenhagen, Denmark

${ }^{4}$ Department of Cardiology, Bispebjerg University Hospital, Copenhagen, Denmark

${ }^{5}$ Clinical Medicine, University of Copenhagen, Copenhagen, Denmark

${ }^{6}$ Department of Cardiology, Odense University Hospital, Odense, Denmark

${ }^{7}$ Department of Cardiology, Roskilde Hospital, Roskilde, Sjaelland, Denmark

${ }^{8}$ Department of Cardiology, Nephrology and Endocrinology, Nordsjaellands Hospital, Hilleroed, Denmark

Contributors The study was designed by RBH and Kl. Data collection was handled by RBH, SH, MP-H, TE, MH, FP, MS, HM, HE, RS and LK. KI and RBH did the data analyses. RBH and KI wrote the first manuscript. All authors critically revised the manuscript and agree to be accountable for all aspects of the work.

Funding The authors have not declared a specific grant for this research from any funding agency in the public, commercial or not-for-profit sectors.

Competing interests None declared.

Patient consent for publication Not required.

Ethics approval The study complied with the Declaration of Helsinki II. The project was approved by the Danish Data Protection Agency (2014-41-3261) and The Danish Medicines Authority (3-3013-987/1), who determined no patient consent was necessary under Danish law.

Provenance and peer review Not commissioned; externally peer reviewed.

Data availability statement Data are available on reasonable request to the corresponding author (RBH).

Open access This is an open access article distributed in accordance with the Creative Commons Attribution Non Commercial (CC BY-NC 4.0) license, which permits others to distribute, remix, adapt, build upon this work non-commercially, and license their derivative works on different terms, provided the original work is properly cited, appropriate credit is given, any changes made indicated, and the use is non-commercial. See: http://creativecommons.org/licenses/by-nc/4.0/.

ORCID iD

Rasmus Bo Hasselbalch http://orcid.org/0000-0003-4274-6268

\section{REFERENCES}

1 lung B, Vahanian A. Epidemiology of acquired valvular heart disease. Can J Cardiol 2014;30:962-70.

2 lung B, Cachier A, Baron G, et al. Decision-Making in elderly patients with severe aortic stenosis: why are so many denied surgery? Eur Heart J 2005;26:2714-20.

3 Baumgartner H, Falk V, Bax JJ, et al. 2017 ESC/EACTS guidelines for the management of valvular heart disease. Eur Heart $J$ 2017;38:2739-91.

4 Nishimura RA, Otto CM, Bonow RO, et al. 2014 AHA/ACC guideline for the management of patients with valvular heart disease: a report of the American College of Cardiology/American heart association Task force on practice guidelines. J Thorac Cardiovasc Surg 2014;148:e1-132.

5 Opolski MP, Staruch AD, Jakubczyk M, et al. CT Angiography for the Detection of Coronary Artery Stenoses in Patients Referred for 
Cardiac Valve Surgery: Systematic Review and Meta-Analysis. JACC Cardiovasc Imaging 2016;9:1059-70.

6 Hasselbalch RB, Engstrøm T, Pries-Heje M, et al. Coronary risk stratification of patients undergoing surgery for valvular heart disease. Int J Cardiol 2017;227:37-42.

7 Catalán P, Leta R, Hidalgo A, et al. Ruling out coronary artery disease with noninvasive coronary multidetector $\mathrm{CT}$ angiography before noncoronary cardiovascular surgery. Radiology 2011;258:426-34.

8 Lobotessi H, Karoussou A, Neofotistou V, et al. Effective dose to a patient undergoing coronary angiography. Radiat Prot Dosimetry 2001;94:173-6.

9 Budoff MJ KR, Ahmadi N, Nasserian C, et al. 64-slice computed tomographic angiography for the diagnosis of intermediate risk coronary artery disease: an evidence-based analysis. Ont Health Technol Assess Ser 2010;10:1-44.

10 Westwood M, Al M, Burgers L, et al. A systematic review and economic evaluation of new-generation computed tomography scanners for imaging in coronary artery disease and congenital heart disease: Somatom definition flash, Aquilion one, brilliance iCT and discovery CT750 HD. Health Technol Assess 2013;17:1-243.

11 Statens Serum Institut. Prices of procedures (Takstsystem) 2014.

12 Dorenkamp M, Bonaventura K, Sohns C, et al. Direct costs and cost-effectiveness of dual-source computed tomography and invasive coronary angiography in patients with an intermediate pretest likelihood for coronary artery disease. Heart 2012;98:460-7.

13 Kreisz FP, Merlin T, Moss J, et al. The pre-test risk stratified cost-effectiveness of 64-slice computed tomography coronary angiography in the detection of significant obstructive coronary artery disease in patients otherwise referred to invasive coronary angiography. Heart Lung Circ 2009;18:200-7.
14 Lee S-P, Jang EJ, Kim Y-J, et al. Cost-Effectiveness of coronary CT angiography in patients with chest pain: comparison with myocardia single photon emission tomography. J Cardiovasc Comput Tomogr 2015;9:428-37.

15 Goverment offices of Sweden. Swedish agency for health technology assessment and assessment of social services (Statens beredning för medicinsk och social utvärdering). coronary angiography for suspected coronary disease (Datortomografi för misstänkt kranskärlssjukdom). SBU Alert-Rapport; 2011.

16 R Core Team. R: a language and Eviroment for statistical computing 2016.

17 Nkomo VT, Gardin JM, Skelton TN, et al. Burden of valvular heart diseases: a population-based study. Lancet 2006;368:1005-11.

18 D'Agostino RS, Jacobs JP, Badhwar V, et al. The Society of Thoracic Surgeons Adult Cardiac Surgery Database: 2019 Update on Outcomes and Quality. Ann Thorac Surg 2019;107:24-32.

19 Larsen LH, Kofoed KF, Dalsgaard M, et al. Assessment of coronary artery disease using coronary computed tomography angiography in patients with aortic valve stenosis referred for surgical aortic valve replacement. Int J Cardiol 2013;168:126-31.

20 Tavakol M, Ashraf S, Brener SJ. Risks and complications of coronary angiography: a comprehensive review. Glob J Health Sci 2012;4:65-93.

21 Rasmussen LA, Bøtker HE, Jensen LO, et al. Quality assurance of the Western Denmark heart registry, a population-based healthcare register. Dan Med J 2017;64:A5414.

22 Schulz-Schüpke S, Helde S, Gewalt S, et al. Comparison of vascular closure devices vs manual compression after femoral artery puncture: the ISAR-CLOSURE randomized clinical trial. JAMA 2014;312:1981-7.

23 Moss AJ, Williams MC, Newby DE, et al. The updated NICE guidelines: cardiac CT as the first-line test for coronary artery disease. Curr Cardiovasc Imaging Rep 2017;10:15. 